Authors' reply: As a general rule, it would seem prudent for one inclined to critique others for repeating points made elsewhere to double-check the originality of one's own complaints. Several of the points raised by Cohen \& Lee have been made before including in this journal (Edgerton \& Cohen, 1994) - and have already been addressed by WHO-affiliated researchers (Sartorius, 1992; Jablensky \& Sartorius et al, 1994).

More pertinently, Cohen \& Lee misunderstand the nature of a 're-analysis' of existing data. They call for closer attention to methodological and cultural factors that might influence outcome measures. We concur, and said so in our article (Craig et al, 1997; p. 232), but the data at our disposal did not permit such an analysis. Instead, our intent was twofold. First, spurred by our own reading of the anthropological literature (Hopper, 1991), as well as by Edgerton \& Cohen's earlier critique, we sought to determine whether the results of the short-term follow-up can suggest other ways of classifying settings than the developed $v$. developing classification. Second, we sought to apply a new methodological tool to see whether it would enable us to formulate better, more refined, questions. Our article reported results on both these counts. Our findings support the wisdom of seeking other ways of describing cultures in order to explain differential outcome in schizophrenia. They also indicate that new ways of analysing data can convey new and important insights. The empirically driven recursive partitioning method we used is quite different from traditional modeldriven approaches. That results from the two methods exhibit a fair degree of concordance, we would argue, is a strength rather than a 'minor modification'. Moreover, our approach allows us to formulate much more specific attributions and interactive effects for predictive variables (p. 231), of precisely the sort Cohen \& Lee call for.

Their most breathtaking complaint is that we fail to consider recent work demonstrating the possibility that a diagnostic grouping (non-affective recurring psychosis) may prevail in the developing countries. In the first place, this is not, to our knowledge, an accepted nosologic entity, or one that local clinicians were asked to identify in DOSMD at that time. Second, this specific diagnostic category is dependent upon a knowledge of course, and thus would have been hopelessly confounded with our dependent variable, and third, although an early paper used a clever bit of epidemiological sleuthing to argue for its potential applicability (Susser \& Wanderling, 1994), Cohen $\&$ Lee appear to take us to task for the preternatural sin of failing to address an argument that appeared in the literature after our own was in press.

A final point that needs response concerns classification. To begin with, there is no sacred grove of permissible categories for cross-cultural research. Distinctions mark useful differences; their validity (utility) depends upon the task at hand. Specifically regarding gender, they first criticise us for presuming the cross-cultural validity of gender and then proceed two sentences later, to commit the same productive sin: they allege that the "demographic reality" of gender imbalances - "more men than women (sic)" in Northern India and Nigeria - ought to be integrated into the analysis, an argument that is difficult to make without assuming the reality of its component parts. Furthermore, they have asked why have we not examined the question "why should one expect better outcomes for women in Chandigarh?". The prediction tree displayed in Figure 1 clearly indicates that based on our analysis no such assertion can be made, and that better outcomes for women is predicted only for those women with non-insidious onset in the predominantly developed settings (that clearly exclude Chandigarh and Ibadan). They should also take note that our definition of 'good' and 'poor' outcome differs from that of their 1994 paper.

Craig, T. J., Slegel, C., Hopper, K., et al (1997) Outcome in schizophrenia and related disorders compared between developing and developed countries. A recursive partitioning re-analysis of the WHO DOSMD data. British journal of Psychiatry. 170, 229-233.

Edgerton, R. B. \& Cohen A. (1994) Culture and schizophrenia: the DOSMD challenge. British journal of Psychiotry, 164, 222-231.

Hopper, H. (1991) Some old questions for the new cross-cultural psychiatry. Medical Anthropology Quarterly. 5. 299-330.

Jablensky, A., Sartorlus, N, Cooper, J. E., ot ol (1994) Culture and schizophrenia: criticisms of WHO studies answered. British journal of Psychiatry, 165, 434-436.

Sartorlus, N. (1992) Prognosis for schizophrenia in the third world: A reevaluation of cross cultural research. Culture, Medicine and Psychiatry, 16, 81-84.

Suscer, E. \& Wanderling, J. (1994) Epidemiology of nonaffective acute remitting psychosis: Sex and social-cultural setting. Archives of Generol Psychiotry, 51, 294-301.

T. Craig VA VISN 3, Bronx, New York
C. Siegel, K. Hopper, S. Lin Nathan Kline Institute for Psychiatric Research, Orangeburg, New York

N. Sartorius Department of Psychiatry, University of Geneva, Switzerland

\section{Use of seclusion, restraint and emergency medication}

Sir: Rangecroft et al (1997) report a significant decrease in seclusion and emergency medication during the course of a study which monitored these interventions. They also suggest that patients with a learning disability may respond quickly to the process of seclusion.

In a similar, large-scale study at $\mathrm{St}$ Andrew's Hospital, we have analysed 3071 incidents of emergency interventions over 18 months. We have found results which broadly support some of these findings and also present some interesting differences. As was found by Rangecroft et al there was a steady reduction in the use of seclusion when staff knew that management techniques were being monitored. There was a further dramatic reduction from an average of 69 to 30 seclusions per month following policy changes requiring medical attendance at seclusions. There appeared, however, to be some 'displacement' effect in that while seclusion figures reduced the recorded use of oral and intramuscular medication, in emergency intervention this increased. This finding is in contrast to Rangecroft et al who report a significant reduction in the use of emergency drugs given intramuscularly.

It is interesting that Rangecroft et al find a lower duration of seclusion in this learning disabled population compared with studies of non-learning disabled subjects. In contrast, we found a significantly higher duration of seclusion on learning disability wards compared with other wards. This supports the finding of Mason (1996) that patients with learning disabilities "tended not to respond as well to seclusion as those without a learning difficulty".

Some findings of Rangecroft et al have now been partially replicated in another sample; however, the contrasts pose questions which need to be addressed. These relate in particular to the effect of reducing one type of emergency intervention on the use of other types of intervention (a possible displacement effect) and the benefits of secluding patients with learning disabilities. These areas have been neglected in the past and warrant further exploration. 
Mason, T. (1996) Seclusion and learning disabilities: Research and deduction. The British Journal of Developmental Disabilities, 42. 149-159.

Rangecroft, M. E. H., Tyrer, S. P. \& Berney, T. P. (1997) The use of seclusion and emergency medication in a hospital for people with learning disability. British journal of Psychiatry, I70. 273-277.

H. Sequeira, S. Halstead St Andrews Hospital, Northampton NNI 5DG

\section{Psychopathological syndromes and familial morbid risk of psychosis}

Sir: I wish to comment on the article by Van Os et al (1997). Before using the term "broad schizoaffective psychosis" the authors should consider the possibility that their original group of schizoaffective psychosis is already too broadly defined. Of the 150 patients with psychosis they diagnosed 65 as schizoaffective, giving a figure of $43 \%$ of consecutively admitted patients. When Brockington \& Leff (1976) looked at the diagnosis of schizoaffective psychosis in what would seem to be an identical setting, in 222 consecutively admitted patients they found an incidence of only eight cases diagnosed according to the same Research Diagnostic Criteria (RDC). An almost tenfold increase in the diagnosis needs an explanation.

The RDC acknowledges that schizoaffective disorders could be either variants of affective disorder or schizophrenia, and in fact lays down criteria to further subdivide schizoaffective psychosis into predominantly depressive or manic and predominantly schizophrenic. This distinction is important as Brockington et al (1980) have shown that schizomania is a variant of bipolar disorder and that schizodepressive disorder is often closely related to schizophrenia (Brockington et al, 1978). As no subdivision has been made this should compromise the results. It is possible therefore that the group of schizoaffective disorder which the authors compared with schizophrenia already had a large number of schizophrenics, hence the findings that schizophrenia familial risk was higher in this group. It would certainly be helpful if this information were available.

Adams, R. \&uylor, M. A. (1976) Catatonia, a prospective clinical study. Archives of General Psychiatry, 33. 579-581.

Brockington, I. F., Waimwright, S. \& Kendell, R. E. (1980) Manic patients with schizophrenic or paranoid symptoms. Psychological Medicine, 10. 73-83.
—, Kendell, R. E., Kellet, J. M., et al (1978) Trials of lithium, chlorpromazine and amitriptyline in schizoaffective patients. British Journal of Psychiatry, 133, 162-168.

- Lef, J. P. (1979) Schizoaffective psychosis: definition and incidence. Psychological Medicine, 9, 91-99.

Van Os, J., Marcelis, M. Sham, P., et al (1997) Psychopathological syndromes and familial morbid risk of psychosis. British journal of Psychiatry. 170. 24I-246.

A. Ryan Division of Mood Disorders, Sunnybrook Health Science Centre, 2075 Bayview Avenue, Toronto, Ontario M4N 3M5, Canada

Authors' reply: The likely reason for the higher rate of RDC schizoaffective disorder in our patients is that diagnoses were not only based on cross-sectional, but also on longitudinal (lifetime) psychopathological data. 'Pure' affective and schizophrenic states will become rarer with time as significant proportions of such patients will experience schizophrenic and affective symptoms respectively.

We agree that separate examination of schizodepressive and schizomanic cases would have been interesting and possibly revealing. The sample size, however, was too small to allow for such analyses.

While these diagnostic issues may be important, we would like to stress that the main findings of our paper involved effects that were independent of proband diagnosis.

J. Van Os, M. Marcelis Department of Psychiatry and Neuropsychology, PO BOX 616,6200 MD

Maastricht, The Netherlands

P. Sham, K. Gilvarry, R. Murray Institute of Psychiatry, London

P. Jones Mapperly Hospital, Nottingham

\section{Treatment, outcome and predictors of response in elderly depressed in-patients}

Sir: Heeren et al (1997) reported a naturalistic prospective study of treatment, outcome and predictors of response in elderly depressed in-patients in The Netherlands. Their findings showed a low percentage of full recovery $(33-45 \%)$, with a high rate of partial recovery $(43-66 \%)$, compared with previous studies. They concluded that a combination of inadequate treatment and therapeutic nihilism played a major role. Similar findings are paralleled in the adult service with failure to treat resistant depression adequately (Bridges et al, 1995).
However, there may be other reasons for such a low response in this study. The sample consisted of a wide variety of affective disorders, ranging from adjustment disorder to organic depression. Indeed, the study claimed to exclude patients with 'dementia'. How this was achieved is not clear and notably patients with Mini-Mental State Examination scores as low as five were recorded, with no evidence to suggest that this was a reversible cognitive impairment at follow-up. Thus this group of patients was an exceedingly heterogeneous mix, making it difficult to compare outcomes with previous studies.

If one assumes that the poor response is due to undertreatment, then this could be confirmed by comparing the two subgroups of patients, those treated with 'classic' antidepressants and 'modern' antidepressants. Undertreatment occurred much more frequently in the former group ( $82 v .36 \%$ ) and if their hypothesis is correct, should show a poorer prognosis. No comment was made regarding these data and it would be interesting to analyse this further.

Bridges, P. K., Hodzdiss, A. D. \& Malizia, A. L. (1995)

Practical management of treatment-resistant affective disorders. British journal of Psychiotry, 170. 436-440.

Heeren, T. J., Derkeen, P., Heycop Ton Ham, B. F. v., ot al (1997) Treatment, outcome and predictors of response in elderly depressed in-patients. British fournal of Psychiatry, 170 436-440.

G. Pinner, W. P. Bouman Department of Health Care of the Elderly, University Hospital, Nottingham NG7 2UH

Authors' reply: We thank Pinner \& Bouman for their critical comments and we agree with them that probably not only elderly depressives are the victims of therapeutic reticence. The first point concerns the heterogeneity of the population. The purpose of our study was to include as much as possible a 'normal' clinical population of elderly depressives and not a highly selected sample as usually occurs in clinical trials. Inclusion and exclusion were based on DSM-III-R criteria and not on the scores of the rating scales (these were used as measures of severity), so it could occur that one patient diagnosed with a recurrent major depressive episode had initially a score of five points on the MMSE. Although, unusual for a depressed patient, several factors such as motivation or physical illness may explain such a low score. 